\title{
Utilização de Endoprótese Auto-Expansível (Stent) Introduzida através da Artéria Femoral para Tratamento de Dissecção da Aorta Descendente
}

\author{
José Honório Palma da Fonseca, Enio Buffolo, Antonio Carlos Carvalho, N ikolaus G eisthovel, \\ Dirceu Rodrigues A Imeida, José Augusto Marcondes de Souza, Ivan Paula Machado, Cláudia Maria \\ Rodrigues Alves
}

São Paulo, SP

Objetivo - Apresentar a correção de dissecção da aorta descendente, utilizando stent recoberto com dácron ${ }^{\circledast}$ introduzido através da artéria femoral na sala de hemodinâmica.

Métodos - Quatro pacientes foram submetidos à sedação, anestesia local de ambas regiões inguinais e a heparinização sistêmica, com cateter contendo o stent introduzido, através da artéria femoral comum, previamente dissecada, até a aorta descendente no seu terço médio.

Resultados - A expansão do stent foi realizada no local onde existia a lesão da íntima, diagnosticada por arteriografia e ecocardiograma. A oclusão da falsa luzfoi imediata. O tempo do procedimento foi em média de lhe 30min. A alta hospitalar ocorreu sem complicações.

Conclusão-Este procedimento poderá proporcionar uma melhora substancial nos resultados do tratamento das dissecções da aorta descendente.

Palavras-chave: aneurisma da aorta, dissecção da aorta, stent

\section{Utilization of Self-Expandable Prosthesis (Stent) Introduced Through the Femoral Artery for Treatment of Dissection of the Descending Aorta}

Purpose - We present four cases of dissection of the thoracic descending aorta that underwent treatment with an auto expandable dácron ${ }^{\circledR}$-covered stent.

Methods - The stent was deployed through the femoral artery in the hemodinamic laboratory after sedation, local anesthesia in both inguinal areas and systemic heparinization. The catheter with the stent was introduced through a cutdown in the left femoral artery until the area in the thoracic aorta with the previously diagnosed tear.

Results - The stent was expanded with immediate occlusion of the false lumen. The whole proccedure took 90 minutes and the patients were discharged without complications.

Conclusion - Stent utilization is an important step to improve treatment results in type B aortic dissection.

Key-words: aorta aneurysm, aorta dissection, stent

Arq Bras Cardiol, volume 70 (nº 6), 389-392, 1998

A dissecção aórtica, envolvendo somente a aorta descendente (tipo B), é uma doença que apresenta altas taxas de morbi-mortalidade, pois, na sua maioria, é acometida em pacientes idosos que apresentam doenças associadas, como doença pulmonar obstrutiva crônica, aterosclerose coronária e algum grau de disfunção renal ${ }^{1}$. Devido ao elevado

Escola Paulista de Medicina - UNIFESP

Correspondência: José Honório Palma da Fonseca - Rua Napoleão de Barros, 715 - $3^{\circ}$ - 04024-002 - São Paulo, SP

Recebido para publicação em 13/2/98

Aceito em 26/3/98 risco cirúrgico dessa população, o tratamento tradicional deste tipo de dissecção é clínico, ficando o tratamento cirúrgico reservado aos pacientes que apresentam complicações na sua evolução, como rotura da aorta torácica, expansão da falsa luz, dor persistente e isquemia visceral ${ }^{2}$. A despeito dos grandes avanços, tanto no diagnóstico como na abordagem cirúrgica das dissecções aórticas, é relatada mortalidade superior a 50\% quando esses pacientes são operados na vigência de complicações e em situações de urgência ${ }^{1,2}$. Devido ao alto potencial de complicações na evolução, alguns autores têm preconizado, atualmente, já na fase aguda, 
o tratamento cirúrgico, utilizando diferentes recursos e técnicas novas, com resultados encorajadores ${ }^{3,4}$.

A utilização clínica dos stents aórticos recobertos com dácron ${ }^{\circledR}$ foi iniciada em nossa instituição, durante ato cirúrgico, em substituição à técnica da "tromba de elefante". Durante a parada circulatória total, esta prótese foi colocada no interior da aorta descendente, sob visão direta, ocluindo a fenda (lesão da íntima), após a artéria subclávia esquerda. A partir desses trabalhos, desenvolvemos experimentalmente, a técnica de inserção dos stents na aorta torácica de cães através da artéria femoral ${ }^{5}$.

Nesta publicação relatamos os quatro primeiros pacientes com dissecção aguda da aorta descendente que foram tratados com sucesso, empregando-se stents aórticos autoexpansíveis, inseridos através da artéria femoral e locados na aorta descendente.

\section{Métodos}

Foram submetidos a este tratamento quatro pacientes, com diagnóstico de dissecção aguda da aorta descendente sendo que todos apresentavam dor torácica importante, apesar da medicação anti-hipertensiva adequada recebida. Os dois últimos casos apresentavam quadro clínico mais complexo: além da dor, foi observada queda do hematócrito, com os exames subsidiários demonstrando hematoma de mediastino e hemotórax. A confirmação do diagnóstico, assim como o tamanho e comprimento da prótese a ser utilizada, foi feita baseada em três exames: aortografia, tomografia e Doppler Ecocardiografia transesofágica. Através da aortografia, observaram-se, nos quatro casos, achados semelhantes: lesão na íntima, única, localizada na transição do terço proximal com o terço médio da aorta descendente (fig. 1). A idade variou de 62 a 82 (média de 73) anos. Apresentavam, também, doenças associadas, como doença pulmonar crônica obstrutiva severa ${ }^{2}$, insuficiência aórtica moderada por ectasia ânulo-aórtica ${ }^{1} \mathrm{e}$ alcoolismo ${ }^{1}$.

O stent aórtico, desenvolvido em nossa instituição, foi confeccionado com fios de aço em zigue-zague, tomando uma formação tubulare recobertos com dácron (fig. 2). Estes cilindros apresentam alta resistência ao colapso radial e

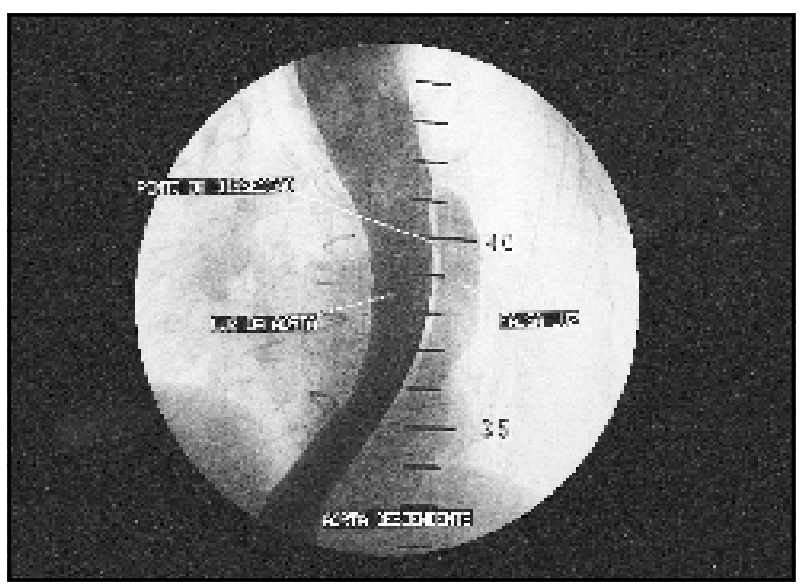

Fig. 1 - Aortografia evidenciando a lesão da íntima na aorta descendente.

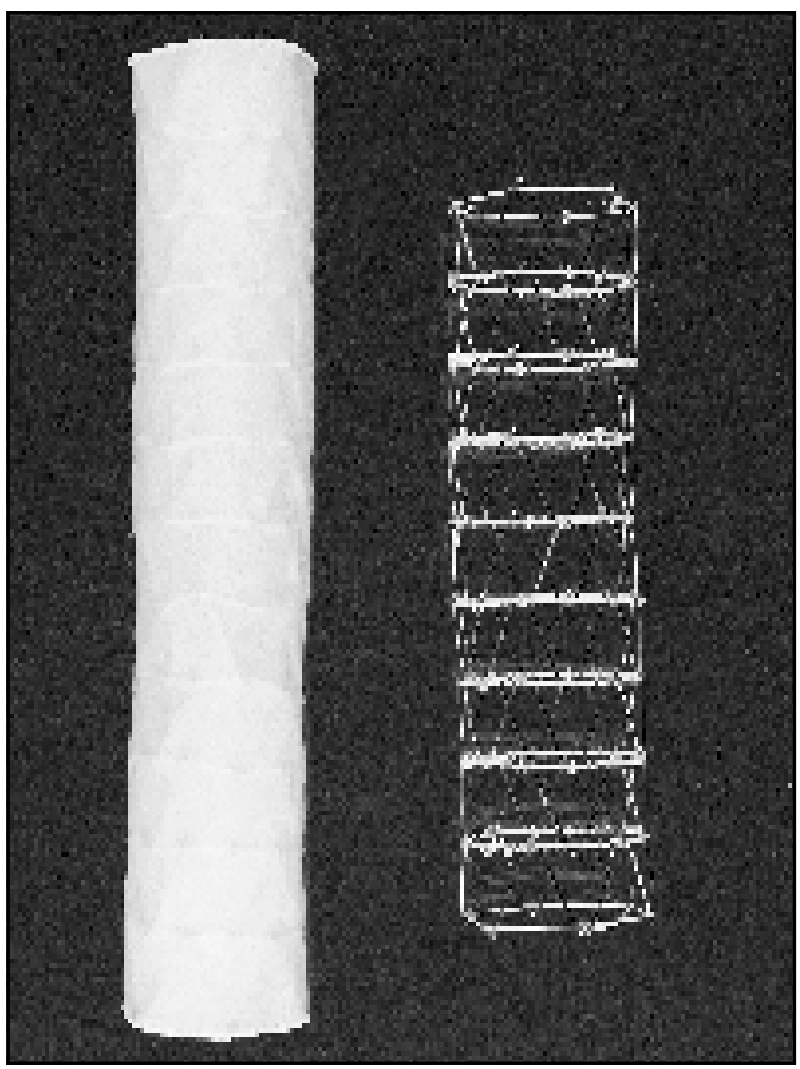

Fig. 2 - Stent aórtico-recoberto com dácron ${ }^{\circledR}$ e só com sua estrutura metálica. Desenvolvido pela Escola Paulista de Medicina e produzido pela Braile Biomédica.

mantêm a capacidade de retornar ao diâmetro originário. Estas próteses foram cuidadosamente comprimidas e inseridas em um cateter com diâmetro $22 \mathrm{~F}$, apresentando $8 \mathrm{~cm}$ de extensão e $3 \mathrm{~cm}$ de diâmetro após sua expansão total, que é feita por uma simples mobilização da bainha.

Na sala de hemodinâmica, sob anestesia local e sedação, realizamos uma nova arteriografia em duas projeções (póstero-anterior e oblíqua anterior esquerda) para confirmar os achados anteriores. Após aortografia, selecionamos a artéria femoral, menos tortuosa, para se introduzir o cateter que contém o stent. A artéria femoral foi, cirurgicamente, isolada e aberta com uma incisão transversa. Utilizamos heparinização (5000U) plena e, sob visão radioscópica, o cateter, contendo o stent e o fio guia, foi introduzido na artéria femoral e avançado, lentamente, até o local escolhido na aorta torácica. O local exato da liberação foi marcado por uma régua radiopaca, colocada no dorso do paciente, antes do início do procedimento. Imediatamente antes da liberação do stent foram administrados nitroprussiato de sódio, pela via endovenosa, para manter a pressão arterial média entre $50 \mathrm{e} 60 \mathrm{mmHg}$ com a finalidade de reduzir o risco de migração do stent, após sua liberação. Observado o posicionamento adequado, o stent auto-expansível foi lentamente liberado por retração da bainha protetora.

\section{Resultados}

Após a liberação do stent e sua completa expansão, realizamos nova aortografia de controle com o cateter pas- 


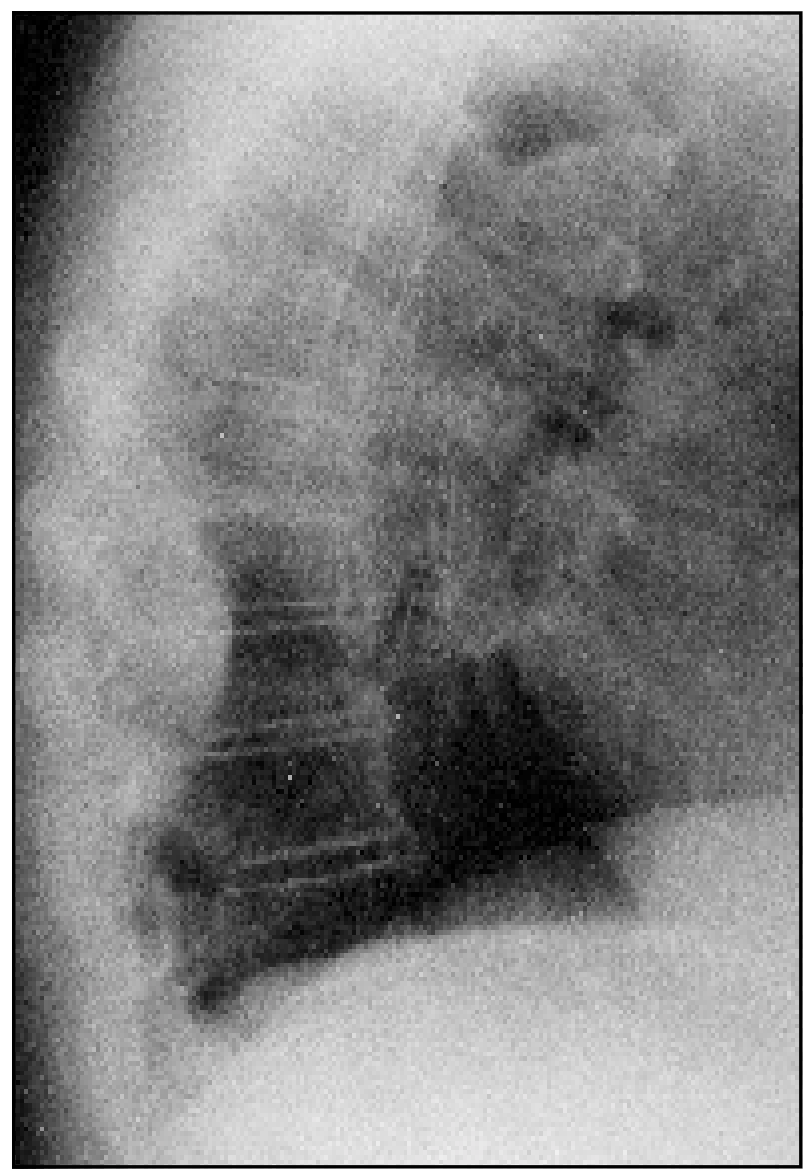

Fig.3 - Radiografia do tórax em perfil onde se observa o stent posicionado na projeção da aorta descendente.

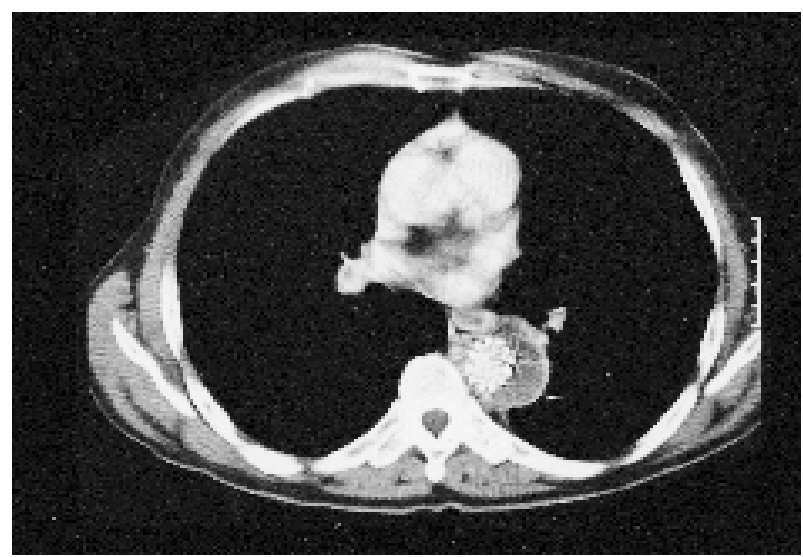

Fig.4 - Tomografia do tórax com contraste evidenciando o stent e a trombose da falsa luz.

sando através da prótese, que demonstrou oclusão total da fenda com trombose da falsa luz nos quatro pacientes.

Os pacientes apresentaram boa evolução com desaparecimento da dor torácica. A alta hospitalar ocorreu em $48 \mathrm{~h}$ em dois pacientes e em 20 dias nos outros dois. Duran-
Endoprótese auto-expansível na dissecção da aorta descendente

te a evolução pós-operatória, até a presente data, os pacientes apresentam-se assintomáticos com radiografias de tórax demonstrando as próteses bem posicionadas (fig. 3). Tomografias de controle feitas ainda na fase aguda, evidenciaram a falsa luz completamente trombosada em todos os casos (fig. 4).

Os dois últimos pacientes desenvolveram quadro febril após a implantação do stent, sendo um atribuído, provavelmente, a reação inflamatória no local de inserção do stent e, outro, devido à infecção na incisão da ingüinotomia. Não observamos ocorrência de hemólise e não utilizamos sangue em nenhum dos pacientes.

\section{Discussão}

O conceito de correção de aneurismas, através da introdução percutânea de uma prótese intraluminar, foi proposto originalmente por Dotter ${ }^{6} \mathrm{em} 1969$, porém, foi Parodi e $\mathrm{col}^{7}$, em 1991, que difundiram esse conceito, tratando pioneiramente, com sucesso, uma série de pacientes com aneurisma de aorta abdominal. Utilizando uma prótese intraluminal expandida por balão, esses autores demostraram que o procedimento é factível e de baixo risco. Dake e $\mathrm{col}^{8}$ demonstraram, pela primeira vez, a possibilidade de tratar os aneurismas da aorta torácica descendente com a introdução, através da artéria femoral, de uma prótese endovascular auto-expansível. Nessa série, trataram dois pacientes com dissecção crônica, sendo que um teve que ser operado na evolução, por continuar com expansão da falsa luz. Inoue e $\operatorname{col}^{9}$ relataram um caso onde uma dissecção de aorta do tipo B foi tratada com uma prótese endovascular, obliterando completamente a fenda e obtendo a trombose da falsa luz.

Nestes quatro pacientes não tivemos dificuldades em progredir e posicionar o stent no local previamente estabelecido e a aortografia realizada, após o procedimento, demonstrou fechamento completo da fenda, a trombose da falsa luz, nenhuma evidência de migração da prótese e, principalmente, a resolução do quadro clínico.

A evolução destes casos demonstra a possibilidade de se poder utilizar o stent aórtico, inserido na sala de hemodinâmica, para tratamento de alguns pacientes selecionados com dissecção aguda da aorta torácica descendente. Entretanto, uma casuística maior, um seguimento a longo prazo maior e o desenvolvimento de cateteres mais adequados serão necessários para que se possa aplicar extensivamente este procedimento no manuseio das dissecções da aorta torácica. Não temos dúvida em afirmar, no entanto, que esta experiência inicial é uma fresta no tempo, permitindo antever um futuro brilhante para as intervenções terapêuticas hemodinâmicas em doenças da aorta descendente.

\section{Referências}

1. Borst HG, Heinemann MK, Stone CD (eds) - Surgical Treatment of Aortic Dissection. New York: Churchill Livingstone, 1996.

2. Miller DC - The continuing dilemma concerning medical versus surgical managemen of patients with acute type B dissections. Semin Thoracic Cardiovasc Surg 1993; 5: 33-5.

3. Glower DD, Fann JI, Speir RHet al - Comparison of medical and surgical therapy 
for uncomplicated descending aortic dissection. Circulation 1990; 52:(suppl IV): IV39-IV46

4. Yoshida H, Yasuda K, Tanabe T - New approach to aortic dissection: development of an insertable aortic prosthesis. Ann Thorac Surg 1994; 58: 806-10.

5. Palma JH, AlmeidaDR, Carvalho AC, Andrade JCS, BuffoloE-Surgical treatment of acute type B aortic dissection using an endoprosthesis (Elephant trunk). Ann Thorac Surg 1997; 63: 1081-4.

6. Dotter CT - Transluminally-placed coilspring endoarterial tube grafts:Long- term patency in canine popliteal artery. Invest Radiol 1969; 4: 329-32.

7. Parodi JC-Endovascular repair of abdominal aortic aneurysms and other arterial lesions. J Vasc Surg 1995; 21: 549-57.

8. Dake MD, Miller DC, Semba CP, Mitchel RS, Walker PJ, Liddell RP Transluminal placement of endovascular stent-grafts for the treatment of descending thoracic aortic aneurysms. N Engl J Med 1994; 331: 1729-34.

9. Inoue K, Sato M, Iwase T et al-Clinical endovascular placement of branched graft for type B aortic dissection. J Thorac Cardiovasc Surg 1996; 112: 1111-3. 